\section{Propuesta de Programa de Atención Integral a la Salud de la Mujer com Enfermedad Cardiovascular de acuerdo com el ciclo reproductivo}

\section{Proposal of Program for Integral Attention of Health in Women with Cardiovascular Disease according to the reproductive cycle}

Hospital Ginecobstétrico "Ramón González Coro" 1

1 Calle 21 entre 4 y 6 Municipio Plaza de la Revolución Ciudad de La Habana. Teléfono: 55 2611-13 E-mail: hgcoro@infomed.sld.cu

\section{Introducción}

A finales del milenio las causas de mortalidad por enfermedad cardiovascular demostraba con cifras elocuentes que la mujer, a la que tradicionalmente se le había atribuido una menor afectación, había alcanzado e incluso superado al hombre en el rubro de enfermedad isquémica e HTA y duplicaba las tasas de este en aspectos como la enfermedad reumática crónica y los trastornos de los lípidos, con aumento de los factores de riesgo para estas enfermedades.

Esto nos hace pensar que se hace necesario modificar algunos de los programas de salud establecidos, desde la concepción de Salud Integral, abarcando la condición psicosocial y de género y teniendo en cuenta las diferentes etapas del ciclo vital con un enfoque clínico-epidemiológico y social.

Esto significa la necesidad de poner en marcha un programa que contemple los intereses de niñas, adolescentes, mujeres fértiles, gestantes y puérperas, mujeres pre y postmenopáusicas, con un esquema de continuidad y no fraccionándolo entre las distintas especialidades y programas. La medicina basada en la evidencia demuestra que una cooperación estrecha y temprana entre todos permitirá obtener mejores resultados y pronósticos en la salud femenina.

Hace más de un siglo una triple sentencia pesaba sobre las cardiópatas: ... soltera, no te cases; casada no tengas hijos; madre no lactes...

Este principio discriminatorio para el sexo femenino está ampliamente superado, pues se ha demostrado que el desarrollo de actividades laborales, la sexualidad, la gestación y la lactancia, en fin, la capacidad de desarrollar una vida plena, puede coexistir con la mayoría de las enfermedades conocidas, incluyendo las cardiopatías.

Se considera que la cardiopatía en el embarazo es la primera causa de morbimortalidad materna no obstétrica. Esta asociación se estima en cifras que fluctúan entre 0,2 y $3,7 \%$, según diferentes autores, a pesar de los avances en el tratamiento de las cardiopatías congénitas y la prevención de la cardiopatía reumática.

La asociación entre cardiopatía y complicaciones del embarazo, como la toxemia, las infecciones y la hemorragia, puede ensombrecer el pronóstico materno-fetal y, eventualmente, ser causa de muertes. La mortalidad materna en general entre las cardiópatas es de alrededor del 3\%, y está en relación con la capacidad funcional.

En el embarazo, además del riesgo de muerte, pueden producirse abortos espontáneos, partos prematuros o retardo del crecimiento intrauterino en el $15 \%$ de las madres cardiópatas, por lo que se necesita un trabajo coordinado y conjunto del obstetra, el cardiólogo y el internista.

El problema principal para estas pacientes lo comportan las prácticas, comportamientos y conductas de riesgo, producto de la información insuficiente de que disponen las pacientes o de bajas percepciones de riesgo y también son el resultado de las prácticas paternalistas que, con el pretexto de la beneficencia, llevan a cabo algunos médicos.

Para brindar servicios de calidad a este grupo de riesgo es necesario ejecutar una serie de acciones que incluyen una política adecuada, organización de los servicios y capacitación de los prestadores de salud, al igual que de las pacientes. 


\section{Objetivos}

\section{Objetivo general}

Incrementar la cobertura y mejorar la atención de las pacientes portadoras de enfermedad cardiovascular, de acuerdo con un enfoque de género, que nos permita contribuir a la elevación de su calidad de vida, redimensionando los recursos disponibles en el Sistema Nacional de Salud, con la participación activa de las pacientes, la familia, la comunidad y la cooperación intersectorial.

\section{Objetivos específicos}

Lograr la atención diferenciada integral de las pacientes con cardiopatía, de acuerdo al ciclo reproductivo.

Identificar y atender precozmente a las pacientes con cardiopatías, para disminuir los riesgos reproductivos a que se hallan expuestas.

Identificar y atender precozmente a las pacientes portadoras de cardiopatías y sus familias en las que se sospechen conductas de riesgo.

Desarrollar acciones de promoción de salud y prevención dirigidas a modificar las causas de morbilidad y mortalidad en este grupo, como parte de los derechos reproductivos, en el marco de las iniciativas por una maternidad sin riesgos.

Perfeccionar y desarrollar los conocimientos de profesionales, técnicos y demás trabajadores del Sistema de Salud sobre la atención a la salud de las pacientes cardiópatas, basándose en las mejores evidencias científicas.

Promover el diseño y ejecución de investigaciones sobre la salud de la mujer con enfermedad cardiovascular, de acuerdo con el ciclo reproductivo, con especial énfasis en la mujer fértil y la embarazada.

Estimular la participación intersectorial, comunitaria y de la familia para mejorar la salud integral en las adolescentes.

\section{Límites}

Este programa será aplicable en todas las instancias del Sistema Nacional de Salud, aunque en sus inicios, su proyección tendrá una mayor significación para las provincias de La Habana y Ciudad Habana, quedando establecida como un programa de pilotaje y control, que establezca la adecuada retroalimentación para su continuo perfeccionamiento. Teniendo en cuenta que el Servicio de Cardiopatía y Embarazo, surge como promotor y centro de referen- cia nacional, se recrea un programa específico para la atención de la gestante con patología cardiovascular asociada, que incluye el establecimiento de una "Guía de práctica clínica para la atención de la gestante con cardiopatía".

Su implementación a partir de su aprobación deberá ser inmediata y los reajustes necesarios se realizarán en un período entre uno y dos años.

\section{Organización y funciones}

La Dirección Nacional de Atención Materno Infantil y Planificación Familiar tendrá a su cargo la responsabilidad de integrar, aplicar, asesorar, controlar y evaluar el programa, siendo el máximo responsable del desarrollo del mismo el Ministro de Salud Pública.

El Viceministerio de Asistencia Médica y Social deberá establecer las coordinaciones para su integración.

El Viceministerio de Docencia e Investigaciones deberá introducir los contenidos relacionados con la atención de la cardiopatía según el género y el ciclo reproductivo en la educación de pre y post-grado, la información científico técnica y las investigaciones médicas.

La Dirección Nacional de Estadísticas estructurará y proveerá la información estadística continua necesaria para el seguimiento y evaluación del programa.

Este esquema se adecuara a los niveles provinciales y municipales.

Las unidades ejecutoras son el Instituto de Cardiología y Cirugía Cardiovascular y el Hospital "Ramón González Coro".

Deberan apoyar este programa: Grupo Nacional de Obstetricia y Ginecología; Grupos Nacionales de Medicina Interna y Medicina General Integral; Grupo Nacional de Cardiología; Grupo Nacional de Pediatría; Grupo Nacional de Psicología; Grupo Nacional de Genética; Sociedad Cubana para el Desarrollo de la Familia (SOCUDEF); Centro Nacional de Educación Sexual (CENESEX).

\section{Actividades}

\section{Organizativas}

Garantizar que esta información esté disponible en las unidades ejecutoras, que deberán realizar el diagnóstico de la morbilidad y mortalidad de la población incluida en este programa. Esto requiere de una actualización del registro de enfermedades crónicas cardiovasculares en los consultorios del 
médico de familia, enfatizando en las adolescentes y mujeres en edad fértil que las padecen.

Se organizaran los recursos humanos y materiales disponibles en las unidades para brindar esta atención integral, con especial interés en el funcionamiento de la consulta de riesgo preconcepcional.

Se desarrollaran equipos de salud multidisciplinarios que incluyan a las especialidades de Pediatría, Obstetricia y Ginecología, Medicina Interna, Medicina General Integral y Cardiología que contarán con el apoyo de las especialidades de Ecocardiografía, Genética, Nutrición, Anestesia, Farmacología, Psicología Clínica y Estomatología.

En las unidades de atención secundaria se programaran consultas dedicadas a la atención de estas pacientes.

Se crearan los servicios especializados de Cardiopatía y Embarazo en las unidades correspondientes, según las condiciones de las unidades hospitalarias, mediante la reorganización y adecuación de los recursos materiales y humanos ya existentes. Se considerará como Servicio Central el radicado en el Hospital "Ramón González Coro".

Conocimiento de las leyes y programas vigentes relacionados con la salud reproductiva por parte del personal vinculado a su atención.

\section{Informativas}

Se brindará información acerca de este programa a los decisores políticos, los sectores de la Salud y Educación y a la comunidad en general, utilizando todos los medios disponibles.

El personal que atiende directamente a este grupo de pacientes utilizará técnicas individuales y grupales para brindar información a la población.

\section{Educativas}

Capacitación del personal que labora directamente con el grupo de referencia, enfatizando en temas de promoción de salud, negociación y concertación, género y autoestima.

Los médicos y enfermeras de la familia contribuirán a elevar los conocimientos de las pacientes y sus familias, maestros, profesores y brigadistas sanitarias de la Federación de Mujeres Cubanas (FMC) en relación con la patología cardiovascular.

Aprovechando la existencia de las Casas de Orientación de la FMC se organizarán y realizaran consultas de consejería multidisciplinarias, sobre temas relacionados con el riesgo cardiovascular.

Los servicios de estomatología realizarán ac- ciones de educación con estas pacientes.

\section{Comunicación}

Se utilizarán todos los canales de comunicación para la orientación de las pacientes.

Los Centros de Promoción y Educación para la Salud diseñaran materiales y soportes educativos y coordinaran con los medios de comunicación masivos la posibilidad de espacios dedicados a este tema.

En todos los servicios estarán disponibles materiales educativos sobre aspectos relevantes de la salud para este grupo de pacientes.

\section{Preventivas y asistenciales}

El principio básico a tener en cuenta es que el mejor momento para diagnosticar y tratar una cardiopatía es antes del embarazo.

Los médicos y enfermeras de la familia desarrollaran las acciones de salud con la población de su radio de acción, adecuándolas a las necesidades identificadas, según dispensarización y diagnóstico de salud. Se hará énfasis en la prevención y tratamiento de la fiebre reumática en edades pediátricas, así como en el diagnóstico y tratamiento oportuno de las cardiopatías congénitas en los niños.

Se priorizará la atención de las pacientes con riesgos socio-conductuales relacionados con un medio familiar desfavorable.

Los controles de salud se efectuarán con la periodicidad necesaria.

Se interconsultará con Cardiología a toda paciente con diagnóstico de cardiopatía.

Las pacientes cardiópatas serán registradas y evaluadas periódicamente en la consulta de planificación familiar.

Atención de las portadoras de cardiopatías congénitas en la consulta de Genética.

Los servicios estomatológicos priorizaran la atención curativa y rehabilitadora en las pacientes.

Se orientará y facilitará el uso de anticonceptivos a este grupo poblacional.

De acuerdo con las edades de las pacientes los profesores de Pediatría, Medicina Interna, Medicina General Integral y Ginecología y Obstetricia realizarán las interconsultas y evaluaciones correspondientes.

Se establece la consulta de riesgo preconcepcional como servicio de consejería para las pacientes en particular y la población en general en relación con la patología cardiovascular. 


\section{Docentes e investigativas}

Se organizará y programará la capacitación en el trabajo a profesionales, técnicos y personal que preste estos servicios.

Se ampliaran los contenidos del tema en los estudios de post-grado.

Se darán a conocer las "Guías prácticas para la atención de la gestante con cardiopatía" en todo el Sistema Nacional de Salud.

Se establecerán centros de referencia para la capacitación, organizando y aprovechando las capacidades disponibles en recursos humanos y materiales.

Se propone el desarrollo de futuras líneas de investigación

Se utilizarán las publicaciones científicas existentes para difundir los resultados de las investigaciones y actualizar los conocimientos sobre el tema.

\section{Vigilancia}

La Dirección Nacional Materno Infantil y sus dependencias analizarán periódicamente el número de gestantes con cardiopatía asociada.

La Dirección Nacional Materno Infantil y sus dependencias analizarán periódicamente las interrupciones de embarazo en pacientes con cardiopatía.

La Dirección Nacional Materno Infantil y sus dependencias analizarán periódicamente la morbilidad y mortalidad de las gestantes con cardiopatía asociada.

De igual modo se analizará el número de nuevos casos detectados de cardiopatía reumática en niños y adolescentes.

La Vicedirección de Asistencia Médica analizará la morbilidad y mortalidad de las pacientes con cardiopatía, de acuerdo con el ciclo reproductivo.

\section{Evaluación}

El cumplimiento del Programa se analizara con la periodicidad necesaria en las diferentes instancias del Sistema Nacional de Salud.

Se efectuarán supervisiones periódicas en todos los niveles.

La evaluación constará de los siguientes aspectos:

Capacitación de los grupos básicos de trabajo en la atención a las pacientes en edad fértil en riesgo por enfermedad cardiovascular.

Dispensarización de acuerdo al Programa de Medicina Familiar.

Funcionamiento de la consulta de riesgo preconcepcional y planificación familiar.

Capacitación de médicos y enfermeras de la familia, así como de estomatólogos en la atención a las referidas pacientes.

Existencia de grupos multidisciplinarios integrados por las especialidades relacionadas con esta atención en las unidades hospitalarias.

Servicio o sala de hospitalización en las unidades hospitalarias.

Atención diferenciada en los servicios de aborto y regulación menstrual.

Atención diferenciada en la gestación, el parto y el puerperio.

Lactancia materna.

Grado de satisfacción de las pacientes con los servicios de salud.

Capacitación de las pacientes en la promoción de salud.

Desarrollo de investigaciones para mejorar la salud reproductiva de las pacientes.

\section{Algunos indicadores relevantes del Programa}

Proporción de embarazos en menores de 20 años. Proporción de embarazos en mayores de 35 años.

Tasa de abortos.

Tasa de mortalidad materna.

Tasa de morbilidad materna.

Prevalencia de tabaquismo.

Prevalencia de obesidad/desnutrición.

Morbilidad por patologías asociadas.

Tasa de incidencia de las ITS.

Incidencia de bajo peso fetal.

Incidencia de cardiopatía en el recién nacido.

Incidencia de malformaciones en el recién nacido.

Recebido em 27 de agosto de 2002

Versão final reapresentada em 29 de novembro de 2002

Aprovado em 29 de dezembro de 2002 